\title{
Cyclization of 5-hexynoic acid to 3-alkoxy-2-cyclohexenones
}

Anne T. Hylden, Eric J. Uzelac, Zeljko Ostojic, Ting-Ting Wu, Keely L. Sacry, Krista L. Sacry, Lin Xi and T. Nicholas Jones ${ }^{*}$

\author{
Letter \\ Address: \\ Department of Chemistry, College of St. Benedict, St. John's \\ University, St. Joseph, MN 56374, USA

\section{Email:} \\ T. Nicholas Jones* - tjones@csbsju.edu \\ * Corresponding author \\ Keywords: \\ acyl chlorides; alcohols; alkynes; cyclization; Lewis acids
}

\author{
Beilstein J. Org. Chem. 2011, 7, 1323-1326. \\ doi:10.3762/bjoc.7.155 \\ Received: 03 June 2011 \\ Accepted: 31 August 2011 \\ Published: 23 September 2011 \\ Associate Editor: D. Dixon \\ (c) 2011 Hylden et al; licensee Beilstein-Institut. \\ License and terms: see end of document.
}

\begin{abstract}
The one-pot cyclization of 5-hexynoic acid to produce 3-alkoxy-2-cyclohexenones proceeds in good yields (58-90\%). 3-Hexynoic acid was converted to its acyl chloride with the aid of oxalyl chloride and was cyclized to 3-chloro-2-cyclohexenone upon addition of indium(III) chloride. Subsequent addition of alcohol nucleophiles led to the desired 3-alkoxy-2-cyclohexenones.
\end{abstract}

\section{Findings}

Synthetic applications of 3-alkoxy-2-cyclohexenones toward the pursuit of natural products have been well documented [1-4]. Traditionally, 3-alkoxy-2-cyclohexenones have been prepared from 1,3-cyclohexadiones under a variety of conditions $[5,6]$. We report herein the cyclization of 5-hexynoyl chloride (2) as a complementary approach to the synthesis of 3-alkoxy-2-cyclohexenones 1 (Scheme 1).

The carbocyclization of 5-hexynoic acid was first reported by Tedder in 1957 [7]. Treatment of 5-hexynoic acid with trifluoroacetic anhydride followed by decomposition of the reaction mixture in methanol under reflux yielded 1,3-cyclohexandione in $25 \%$ yield. Later, Smit and coworkers reported the closely related acyclic reaction between alkynes and acyl cations

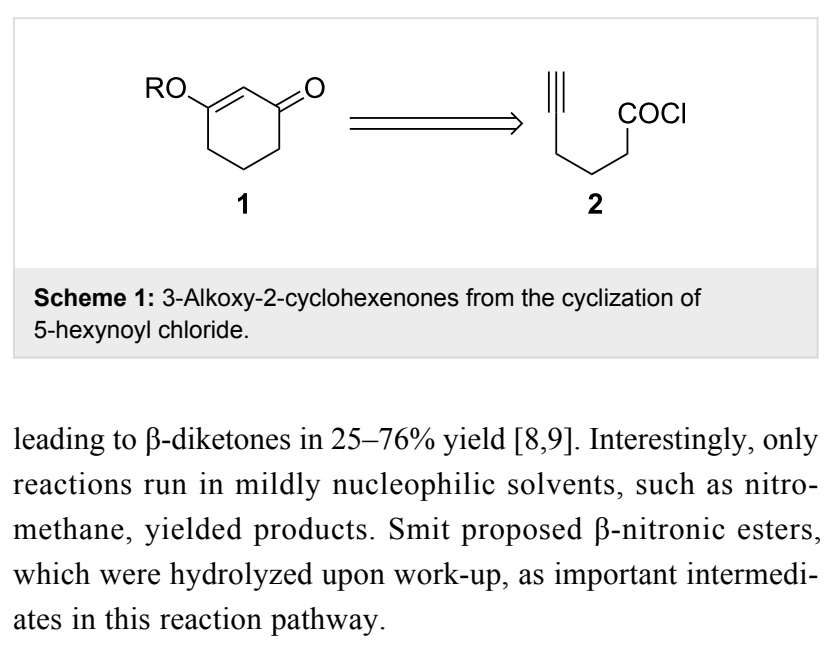


With the work of Tedder and Smit in mind, we began our study with the cyclization of the known 5-hexynoyl chloride (2) [10] under Lewis acid conditions and quenching of the reaction with ethanol (Table 1). Our first cyclization attempt utilized silver tetrafluoroborate, which was found to be ineffective at promoting the cyclization. Use of aluminum chloride led to cyclization, but in very modest yield (26\%). Titanium(IV) chloride was also screened, however no cyclization was observed. A dramatic increase in yield (71\%) was obtained with the aid of indium(III) chloride. Additional metal chloride salts have been screened under our cyclization conditions (Table 1, entries 5-8). Although these salts promoted the desired cyclization, they were not found to be as effective as indium(III) chloride.

Table 1: Cyclization of 5-hexynoyl chloride to 3-ethoxy-2-cyclohexenone under Lewis acid conditions.

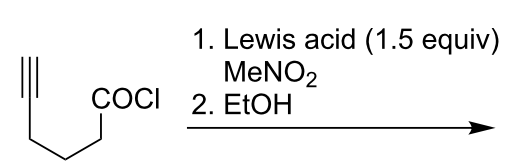

2

\begin{tabular}{ccc}
\hline Entry & Lewis acid & Yield \\
\hline 1 & $\mathrm{AgBF}_{4}$ & n.r. \\
2 & $\mathrm{AlCl}_{3}$ & $26 \%$ \\
3 & $\mathrm{TiCl}_{4}$ & n.r. \\
4 & $\mathrm{InCl}_{3}$ & $71 \%$ \\
5 & $\mathrm{BiCl}_{3}$ & $17 \%$ \\
6 & $\mathrm{CuCl}_{2}$ & $4 \%$ \\
7 & $\mathrm{ZnCl}_{2}$ & $8 \%$ \\
8 & $\mathrm{FeCl}_{3}$ & $63 \%$ \\
\hline
\end{tabular}

$a_{n} . r .=$ no reaction.

We did not observe formation of the alternative 5-membered cyclic product. This result is consistent with those reported by Smit whose acyclic cases led exclusively to acylation at the unsubstituted end of terminal alkynes [8]. Additionally, the preference for formation of the 6-membered cyclization product is likely biased by geometric factors due to the propylene tether linking the acyl chloride and alkyne moieties.

Recently we have developed one-pot conditions for this reaction starting from 5-hexynoic acid (3), see Table 2. ${ }^{1} \mathrm{H}$ NMR analysis of aliquots from the reaction mixture indicated that 3 was completely converted to its acid chloride 2 within 2.5 hours. Indium(III) chloride was added directly to the flask upon formation of acid chloride 2. Significantly, omitting nitromethane as a solvent did not hinder the reaction, indicating that the chloride ion concentration was sufficient to promote the cyclization event. In addition to simplifying the overall process, the yield was also improved from $71 \%$ to $81 \%$ for the ethyl case. Consistent by-products under these conditions were dialkyl oxalates formed from esterification of unreacted oxalyl chloride with excess alcohol nucleophiles, and thus a basic hydrolysis step was added to our work-up procedure. Secondary alcohols required heating to $90{ }^{\circ} \mathrm{C}$ following addition of the alcohol nucleophile to promote product formation. IR and NMR spectral data can be found in Supporting Information File 1.

Table 2: One-pot procedure for the cyclization of 5-hexynoyc acid to 3-alkoxy-2-cyclohexenones.

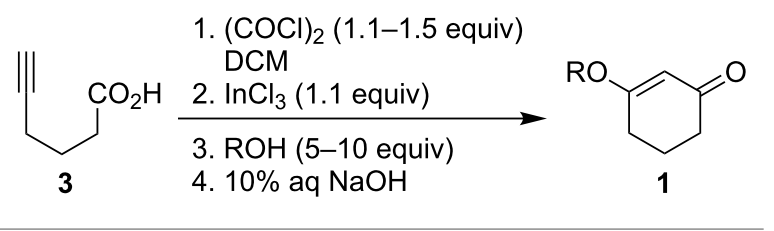

\begin{tabular}{ccc}
\hline Product & $\mathrm{R}$ & Yield \\
\hline 1a & $\mathrm{Et}$ & $81 \%$ \\
1b & $\mathrm{Me}$ & $72 \%$ \\
1c & $n-\mathrm{Pr}$ & $89 \%$ \\
1d & $\mathrm{iPr}$ & $74 \% \mathrm{a}$ \\
1e & $n-\mathrm{Bu}$ & $74 \%$ \\
1f & $s-\mathrm{Bu}$ & $70 \% \mathrm{a}$ \\
1g & $t-\mathrm{Bu}$ & $0 \% \mathrm{~b}$ \\
1h & $\mathrm{Ph}$ & $62 \%$ \\
1i & $\mathrm{Bn}$ & $58 \%$ \\
1j & $\mathrm{CH}_{2}=\mathrm{CH}-\mathrm{CH}_{2}$ & $90 \%$ \\
\hline
\end{tabular}

aRequired heating to $90{ }^{\circ} \mathrm{C}$ following the addition of alcohol nucleophiles to promote product formation. ${ }^{b}$ Only 3-chloro-2-cyclohexenone was observed in the ${ }^{1} \mathrm{H}$ NMR spectrum of the crude reaction mixture.

Additional evidence for the participation of chloride ion in this reaction was given by the isolation of the known 3-chloro-2cyclohexenone (4) [11] from reactions that involved sterically hindered alcohols such as tert-butanol (Table 2). Enone 4 was prepared directly in $78 \%$ yield under our cyclization conditions except that the addition of an alcohol nucleophile was omitted (Scheme 2).

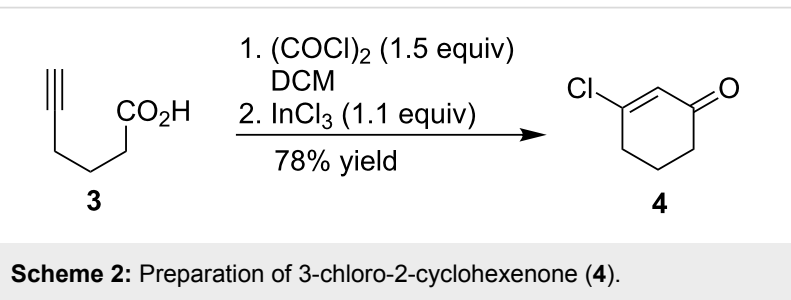

Based on the isolation of $\mathbf{4}$ and the apparent steric influence of the alcohol nucleophiles, as observed in the yield data in Table 2, we have proposed a possible pathway for this process 
(Scheme 3). Acid 3 was converted to the acid chloride 2 by treatment of oxalyl chloride [10]. In situ addition of indium(III) chloride promoted cyclization of 2 to 3-chloro-2-cyclohexenone (4) and was followed by quenching with alcohol nucleophiles, yielding 3-alkoxy-2-cyclohexenones $\mathbf{1}$.

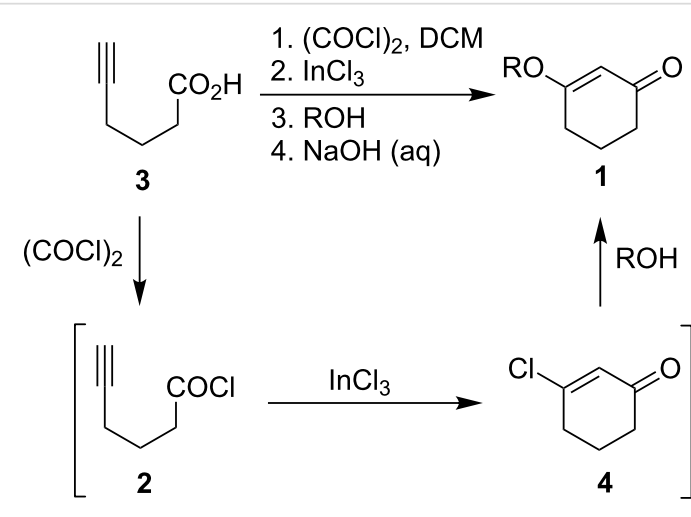

Scheme 3: Proposed pathway for the one-pot conversion of 5-hexynoic acid to 3-alkoxy-2-cyclohexenones.

Related examples by Forsyth [2] and Clausen [4] support our proposed mechanism. They reported conjugate addition of alcohol nucleophiles to 3-chloro-2-cyclohexenone substrates under basic conditions followed by elimination of the chloride. The key difference between their reported conditions and ours is that our cyclizations are Lewis acid mediated.

Building on the work of Tedder [7] and Smit [8,9] we have improved the alkynoic acid cyclization approach in a manner that allows ready access to 3-alkoxy-2-cyclohexenones in good yields. Further elaboration of this methodology is ongoing.

\section{Experimental}

General methods: All experiments were carried out under an atmosphere of dry nitrogen in oven-dried glassware. Solvents, starting materials and reagents were purchased (Sigma-Aldrich and Fisher Scientific) and used without further purification. ${ }^{1} \mathrm{H}$ and ${ }^{13} \mathrm{C}$ NMR spectra were obtained on a Varian Gemini 2000 (300 MHz) spectrometer with $\mathrm{CDCl}_{3}$ as solvent. Infrared spectra were obtained on a Thermo Nicolet 380 FT-IR spectrometer with Smart IR ATR ( $\mathrm{Si}$ or ZnSe). Mass spectra were taken with a Varian $3800 \mathrm{GC} /$ Saturn $2000 \mathrm{MS}$ (ion trap).

3-Methoxy-2-cyclohexenone (1b): 5-Hexynoic acid (3) (0.267 g, $2.38 \mathrm{mmol})$ was combined with oxalyl chloride $(0.396 \mathrm{~g}$, $3.05 \mathrm{mmol})$ in anhydrous dichloromethane $(1.5 \mathrm{~mL})$ in a nitrogen-purged $25 \mathrm{~mL}$ round-bottom flask and stirred for 2.5 hours. Indium(III) chloride ( $0.565 \mathrm{~g}, 2.55 \mathrm{mmol})$ was added and the mixture was stirred for 3 hours. Methanol $(0.401 \mathrm{~g}$, $12.5 \mathrm{mmol}$ ) was then added and the mixture was stirred for
15 minutes. Next, a saturated $\mathrm{NaHCO}_{3}$ solution $(6 \mathrm{~mL})$ and THF ( $3 \mathrm{~mL}$ ) were added and stirred for 15 minutes, followed by addition of $\mathrm{NaOH}(10 \%$ aq, $6 \mathrm{~mL})$ and 5 minutes of stirring. The mixture was extracted with dichloromethane $(3 \times 10 \mathrm{~mL})$ and the combined organic layers were dried over $\mathrm{CaCl}_{2}$ and concentrated in vacuo. Solvent exchange with $\mathrm{CHCl}_{3}(2 \times$ $5 \mathrm{~mL})$ gave the product $(0.216 \mathrm{~g}, 1.71 \mathrm{mmol}, 72 \%)$ as an oil. ${ }^{1} \mathrm{H}$ NMR $\left(300 \mathrm{MHz}, \mathrm{CDCl}_{3}\right) \delta 5.36(\mathrm{~s}, 1 \mathrm{H}, \mathrm{CH}), 3.68(\mathrm{~s}, 3 \mathrm{H}$, $\left.\mathrm{CH}_{3}\right), 2.40\left(\mathrm{t}, J=6.3 \mathrm{~Hz}, 2 \mathrm{H}, \mathrm{CH}_{2}\right), 2.34(\mathrm{t}, J=6.5 \mathrm{~Hz}, 2 \mathrm{H}$, $\left.\mathrm{CH}_{2}\right), 1.97\left(\mathrm{tt}, J=6.3,6.5 \mathrm{~Hz}, 2 \mathrm{H}, \mathrm{CH}_{2}\right) ;{ }^{13} \mathrm{C} \mathrm{NMR}(300 \mathrm{MHz}$, $\mathrm{CDCl}_{3}$ ) $\delta 195.1,174.3,97.6,50.9,31.9,24.0,16.4$; IR (ATR, neat) $2946,1645,1603,1378,1225,1186,1135,1003,826$, $757 \mathrm{~cm}^{-1}$; EIMS $(\mathrm{m} / \mathrm{z}): 127$.

3-Isopropoxy-2-cyclohexenone (1d): 5-Hexynoic acid $(0.241 \mathrm{~g}, 2.15 \mathrm{mmol})$ and oxalyl chloride $(0.361 \mathrm{~g}, 2.84 \mathrm{mmol})$ were combined with anhydrous dichloromethane $(1.5 \mathrm{~mL})$ in a nitrogen-purged $25 \mathrm{~mL}$ round-bottom flask and stirred for 2.5 hours. Indium(III) chloride $(0.522 \mathrm{~g}, 2.36 \mathrm{mmol})$ was added and the mixture was stirred for 3 hours. 2-propanol (1.352 g, $22.5 \mathrm{mmol}$ ) was then added and the mixture was stirred for 15 minutes at $90{ }^{\circ} \mathrm{C}$. The reaction mixture was cooled to room temperature and a saturated $\mathrm{NaHCO}_{3}$ solution $(6 \mathrm{~mL})$ and THF ( $3 \mathrm{~mL}$ ) were added and stirred for 15 minutes, followed by addition of $\mathrm{NaOH}(10 \%$ aq, $6 \mathrm{~mL})$ and 15 more minutes of stirring. The mixture was extracted with DCM $(2 \times 15 \mathrm{~mL})$ and the combined organic layers were dried over $\mathrm{CaCl}_{2}$ and concentrated in vacuo. Solvent exchange with $\mathrm{CHCl}_{3}(3 \times 5 \mathrm{~mL})$ gave the pure product $(0.245 \mathrm{~g}, 1.59 \mathrm{mmol}, 74 \%)$ as an oil. ${ }^{1} \mathrm{H} \mathrm{NMR}$ $\left(300 \mathrm{MHz}, \mathrm{CDCl}_{3}\right) \delta 5.33$ (s, $\left.1 \mathrm{H}, \mathrm{CH}\right), 4.41$ (sept., $J=6.1 \mathrm{~Hz}$, $1 \mathrm{H}, \mathrm{CH}), 2.35$ (t, $\left.J=6.5 \mathrm{~Hz}, 2 \mathrm{H}, \mathrm{CH}_{2}\right), 2.32$ (t, $J=7.1 \mathrm{~Hz}, 2 \mathrm{H}$, $\left.\mathrm{CH}_{2}\right), 1.95\left(\mathrm{tt}, J=6.5,7.1 \mathrm{~Hz}, 2 \mathrm{H}, \mathrm{CH}_{2}\right), 1.27$ (d, $J=6.1 \mathrm{~Hz}$, $\left.6 \mathrm{H}, 2 \mathrm{CH}_{3}\right) ;{ }^{13} \mathrm{C} \mathrm{NMR}\left(\mathrm{CDCl}_{3}\right) \delta 195.4,172.4,98.3,66.2,31.9$, 24.8, 16.6; IR (ATR, neat): 2980, 1645, 1594, 1379, 1219, 1183, 1105, 940, 824, $752 \mathrm{~cm}^{-1}$; EIMS $(\mathrm{m} / \mathrm{z}): 155$.

\section{${ }^{1} \mathrm{H}$ NMR data for compounds 1a, 1c, 1e, 1f,}

\section{$1 \mathrm{~h}-\mathrm{j}$ and 4}

3-Ethoxy-2-cyclohexenone (1a): ${ }^{1} \mathrm{H} \mathrm{NMR}\left(300 \mathrm{MHz}, \mathrm{CDCl}_{3}\right)$ $\delta 5.34(\mathrm{~s}, 1 \mathrm{H}, \mathrm{CH}), 3.89\left(\mathrm{q}, J=7.1 \mathrm{~Hz}, 2 \mathrm{H}, \mathrm{CH}_{2}\right), 2.39$ (t, $J=$ $\left.6.3 \mathrm{~Hz}, 2 \mathrm{H}, \mathrm{CH}_{2}\right), 2.34\left(\mathrm{t}, J=6.5 \mathrm{~Hz}, 2 \mathrm{H}, \mathrm{CH}_{2}\right), 1.97(\mathrm{tt}, J=$ 6.3, $\left.6.5 \mathrm{~Hz}, 2 \mathrm{H}, \mathrm{CH}_{2}\right), 1.35\left(\mathrm{t}, J=7.1 \mathrm{~Hz}, 3 \mathrm{H}, \mathrm{CH}_{3}\right)$.

3-Propoxy-2-cyclohexenone (1c): ${ }^{1} \mathrm{H}$ NMR (300 MHz, $\mathrm{CDCl}_{3}$ ) $\delta 5.33(\mathrm{~s}, 1 \mathrm{H}, \mathrm{CH}), 3.76\left(\mathrm{t}, J=6.5 \mathrm{~Hz}, 2 \mathrm{H}, \mathrm{CH}_{2}\right), 2.39(\mathrm{t}, J=$ $\left.6.3 \mathrm{~Hz}, 2 \mathrm{H}, \mathrm{CH}_{2}\right), 2.32\left(\mathrm{t}, J=6.5 \mathrm{~Hz}, 2 \mathrm{H}, \mathrm{CH}_{2}\right), 1.96(\mathrm{tt}, J=$ 6.3, $6.5 \mathrm{~Hz}, 2 \mathrm{H}, \mathrm{CH}_{2}$ ), 1.73 (tq, $J=6.5,7.1 \mathrm{~Hz}, 2 \mathrm{H}, \mathrm{CH}_{2}$ ), 0.96 (t, $J=7.4 \mathrm{~Hz}, 2 \mathrm{H}, \mathrm{CH}_{3}$ ).

3-Butoxy-2-cyclohexenone (1e): ${ }^{1} \mathrm{H} \mathrm{NMR}\left(300 \mathrm{MHz}, \mathrm{CDCl}_{3}\right)$ $\delta 5.33(\mathrm{~s}, 1 \mathrm{H}, \mathrm{CH}), 3.81\left(\mathrm{t}, J=6.5 \mathrm{~Hz}, 2 \mathrm{H}, \mathrm{CH}_{2}\right), 2.38(\mathrm{t}, J=$ 
$\left.6.3 \mathrm{~Hz}, 2 \mathrm{H}, \mathrm{CH}_{2}\right), 2.32\left(\mathrm{t}, J=6.6 \mathrm{~Hz}, 2 \mathrm{H}, \mathrm{CH}_{2}\right), 1.96(\mathrm{tt}, J=$ $\left.6.3,6.6 \mathrm{~Hz}, 2 \mathrm{H}, \mathrm{CH}_{2}\right), 1.69\left(\mathrm{tt}, J=6.5,7.4 \mathrm{~Hz}, 2 \mathrm{H}, \mathrm{CH}_{2}\right), 1.41$ (tq, $J=7.4 \mathrm{~Hz}, 2 \mathrm{H}, \mathrm{CH}_{2}$ ), $0.93\left(\mathrm{t}, J=7.4 \mathrm{~Hz}, 2 \mathrm{H}, \mathrm{CH}_{2}\right.$ ).

3-(sec-Butoxy)-2-cyclohexenone (1f): ${ }^{1} \mathrm{H}$ NMR $(300 \mathrm{MHz}$, $\left.\mathrm{CDCl}_{3}\right) \delta 5.32(\mathrm{~s}, 1 \mathrm{H}, \mathrm{CH}), 4.19(\mathrm{~m}, 1 \mathrm{H}, \mathrm{CH}), 2.36(\mathrm{t}, J=$ $\left.6.3 \mathrm{~Hz}, 2 \mathrm{H}, \mathrm{CH}_{2}\right), 2.33\left(\mathrm{t}, J=6.6 \mathrm{~Hz}, 2 \mathrm{H}, \mathrm{CH}_{2}\right), 1.96(\mathrm{tt}, J=$ $\left.6.3,6.6 \mathrm{~Hz}, 2 \mathrm{H}, \mathrm{CH}_{2}\right), 1.61\left(\mathrm{~m}, 2 \mathrm{H}, \mathrm{CH}_{2}\right), 1.24(\mathrm{~d}, J=6.2 \mathrm{~Hz}$, $\left.3 \mathrm{H}, \mathrm{CH}_{3}\right), 0.90\left(\mathrm{t}, J=7.5 \mathrm{~Hz}, 3 \mathrm{H}, \mathrm{CH}_{3}\right)$.

3-Phenoxy-2-cyclohexenone (1h): ${ }^{1} \mathrm{H}$ NMR $(300 \mathrm{MHz}$, $\left.\mathrm{CDCl}_{3}\right) \delta 7.37(\mathrm{t}, J=7.6 \mathrm{~Hz}, 2 \mathrm{H}, 2 \mathrm{CH}), 7.22(\mathrm{dd}, J=7.6$, $7.7 \mathrm{~Hz}, 1 \mathrm{H}, \mathrm{CH}), 7.02(\mathrm{~d}, J=7.7 \mathrm{~Hz}, 2 \mathrm{H}, 2 \mathrm{CH}), 5.10(\mathrm{~s}, 1 \mathrm{H}$, $\mathrm{CH}), 2.64\left(\mathrm{t}, J=6.3 \mathrm{~Hz}, 2 \mathrm{H}, \mathrm{CH}_{2}\right), 2.36(\mathrm{t}, J=6.5 \mathrm{~Hz}, 2 \mathrm{H}$, $\left.\mathrm{CH}_{2}\right), 2.06\left(\mathrm{tt}, J=6.3,6.5 \mathrm{~Hz}, 2 \mathrm{H}, \mathrm{CH}_{2}\right.$ ).

3-Benzyloxy-2-cyclohexenone (1i): ${ }^{1} \mathrm{H}$ NMR $(300 \mathrm{MHz}$, $\left.\mathrm{CDCl}_{3}\right) \delta 7.37(\mathrm{~m}, 5 \mathrm{H}, 5 \mathrm{CH}), 5.48(\mathrm{~s}, 1 \mathrm{H}, \mathrm{CH}), 4.89(\mathrm{~s}, 2 \mathrm{H}$, $\mathrm{CH}_{2}$ ), 2.47 (t, $\left.J=6.3 \mathrm{~Hz}, 2 \mathrm{H}, \mathrm{CH}_{2}\right), 2.37$ (t, $J=6.5 \mathrm{~Hz}, 2 \mathrm{H}$, $\left.\mathrm{CH}_{2}\right), 2.00\left(\mathrm{tt}, J=6.3,6.5 \mathrm{~Hz}, 2 \mathrm{H}, \mathrm{CH}_{2}\right)$.

3-Allyloxy-2-cyclohexenone (1j): ${ }^{1} \mathrm{H} \mathrm{NMR}\left(300 \mathrm{MHz}, \mathrm{CDCl}_{3}\right)$ $\delta 5.95(\mathrm{ddt}, J=5.6,9.2,15.4 \mathrm{~Hz}, 1 \mathrm{H}, \mathrm{CH}), 5.38(\mathrm{dd}, J=1.4$, $9.2 \mathrm{~Hz}, 1 \mathrm{H}, \mathrm{CH}), 5.35(\mathrm{~s}, 1 \mathrm{H}, \mathrm{CH}), 5.29(\mathrm{dd}, J=1.4,5.6 \mathrm{~Hz}$, $1 \mathrm{H}, \mathrm{CH}), 4.36\left(\mathrm{~d}, J=5.6 \mathrm{~Hz}, 2 \mathrm{H}, \mathrm{CH}_{2}\right), 2.42(\mathrm{t}, J=6.2 \mathrm{~Hz}, 2 \mathrm{H}$, $\left.\mathrm{CH}_{2}\right), 2.33\left(\mathrm{t}, J=6.6 \mathrm{~Hz}, 2 \mathrm{H}, \mathrm{CH}_{2}\right), 1.97(\mathrm{tt}, J=6.2,6.6 \mathrm{~Hz}$, $\left.2 \mathrm{H}, \mathrm{CH}_{2}\right)$.

3-Chloro-2-cyclohexenone (4): ${ }^{1} \mathrm{H}$ NMR (300 MHz, $\left.\mathrm{CDCl}_{3}\right) \delta$ $6.21(\mathrm{~s}, 1 \mathrm{H}, \mathrm{CH}), 2.68\left(\mathrm{t}, J=6.1 \mathrm{~Hz}, 2 \mathrm{H}, \mathrm{CH}_{2}\right), 2.39(\mathrm{t}, J=$ $\left.6.7 \mathrm{~Hz}, 2 \mathrm{H}, \mathrm{CH}_{2}\right), 2.08$ (tt, $J=6.1,6.7 \mathrm{~Hz}, 2 \mathrm{H}, \mathrm{CH}_{2}$ ).

\section{Supporting Information}

\section{Supporting Information File 1}

IR and NMR data for compounds $\mathbf{1 a}-\mathbf{f}, \mathbf{1 h}-\mathbf{j}$ and $\mathbf{4}$.

[http://www.beilstein-journals.org/bjoc/content/

supplementary/1860-5397-7-155-S1.pdf]

\section{References}

1. Zhao, Y.; Zhou, Y.; Liang, L.; Yang, X.; Du, F.; Li, L.; Zhang, H. Org. Lett. 2009, 11, 555-558. doi:10.1021/ol802608r

2. Demeke, D.; Forsyth, C. J. Tetrahedron 2002, 58, 6531-6544. doi:10.1016/S0040-4020(02)00663-4

3. Hackett, S.; Livinghouse, T. J. Org. Chem. 1986, 51, 879-885. doi:10.1021/jo00356a025

4. Rønnest, M. H.; Rebacz, B.; Markworth, L.; Terp, A. H.; Larsen, T. O.; Krämer, A.; Clausen, M. H. J. Med. Chem. 2009, 52, 3342-3347. doi:10.1021/jm801517j

5. Gannon, W. F.; House, H. O. Org. Synth. 1960, 40, 41.
6. Bhosale, R. S.; Bhosale, S. V.; Bhosale, S. V.; Wang, T.; Zubaidha, P. K. Tetrahedron Lett. 2004, 45, 7187-7188. doi:10.1016/j.tetlet.2004.08.044

7. Ferrier, R. J.; Tedder, J. M. J. Chem. Soc. 1957, 1435-1437.

8. Smit, W. A.; Semenovsky, A. V.; Kucherov, V. F.; Chernova, T. N.; Krimer, M. Z.; Lubinskaya, O. V. Tetrahedron Lett. 1971, 12, 3101-3106. doi:10.1016/S0040-4039(01)97101-1

9. Roitburd, G. V.; Smit, W. A.; Semenovsky, A. V.; Shchegolev, A. A.; Kucherov, V. F.; Chizhov, O. S.; Kadentsev, V. I. Tetrahedron Lett. 1972, 13, 4935-4940. doi:10.1016/S0040-4039(01)94470-3

10. Earl, R. A.; Vollhardt, K. P. C. J. Org. Chem. 1984, 49, 4786-4800. doi:10.1021/jo00199a009

11. Piers, E.; Grierson, J. R.; Lau, C. K.; Nagakura, I. Can. J. Chem. 1982, 60, 210-223. doi:10.1139/v82-033

\section{License and Terms}

This is an Open Access article under the terms of the Creative Commons Attribution License

(http://creativecommons.org/licenses/by/2.0), which permits unrestricted use, distribution, and reproduction in any medium, provided the original work is properly cited.

The license is subject to the Beilstein Journal of Organic Chemistry terms and conditions:

(http://www.beilstein-journals.org/bjoc)

The definitive version of this article is the electronic one which can be found at: doi:10.3762/bjoc. 7.155 